\title{
Prevalência de sintomas de disfunção temporomandibular, fatores associados e impacto sobre a qualidade de vida em usuários da rede de atenção primária à saúde
}

Prevalence of symptoms of temporomandibular disorders, associated factors and impact on quality of life in users of the primary healthcare network

Prevalencia de síntomas de trastornos temporomandibulares, factores asociados e impacto em la calidad de vida em usuarios de la red de atención primaria de salud

Vitória Lúcio Henrique

ORCID: https://orcid.org/0000-0002-8580-7422 Universidade Federal de Alagoas, Brasil E-mail: vitoria.henrique@ foufal.ufal.br

Kaio Coura Melo Pacheco

ORCID: https://orcid.org/0000-0001-7804-5426 Universidade Federal de Alagoas, Brasil

E-mail:kaio.melo_95@hotmail.com

Igor Hudson Albuquerque e Aguiar

ORCID: https://orcid.org/0000-0001-8914-7452 Universidade Federal de Alagoas, Brasil

E-mail:igor.aguiar@foufal.ufal.br

Wanessa Carmen de Oliveira Brito

ORCID: https://orcid.org/0000-0003-1167-2905 Universidade Federal de Alagoas, Brasil E-mail:wanessa.brito@ foufal.ufal.br Pâmela Lopes Pedro da Silva

ORCID: https://orcid.org/0000-0003-4537-6821 Universidade Federal da Paraíba, Brasil E-mail: pamelapan_lopes@hotmail.com André Ulisses Dantas Batista

ORCID: https://orcid.org/0000-0003-1593-0174 Universidade Federal da Paraíba, Brasil E-mail: andreulisses@yahoo.com.br

George Azevedo Lemos

ORCID: https://orcid.org/0000-0002-2140-216X Universidade Federal de Alagoas, Brasil E-mail: george.lemos@icbs.ufal.br

\section{Resumo}

Introdução: Estudos epidemiológicos demonstram elevada prevalência de sinais e/ou sintomas de Disfunção Temporomandibular (DTM) na população adulta. Contudo, poucos estudos avaliaram esta disfunção na atenção primária à saúde. Objetivo: Avaliar prevalência de sintomas de DTM, fatores associados e, impacto sobre qualidade de vida em usuários da rede de atenção primária à saúde da cidade de Maceió/AL. Método: Avaliou-se 108 participantes quanto a presença de sintomas de DTM (índice anamnésico DMF),sintomas sugestivos de ansiedade e depressão (escala Hospital Anxiety and Depression- HAD), e sintomas sugestivos de estresse (Inventário de Sintomas de Stress para Adultos - ISSL); além da qualidade de vida relacionada a saúde oral, avaliada pela versão resumida do Oral Health-related (OHIP-14). Resultados: 55,6\% apresentaram sintomas de DTM e 19,4\% exibiram necessidade de tratamento. O sexo feminino e o autorrelato de hábitos parafuncionais foram preditores para os sintomas de DTM, e o estresse e hábitos para a necessidade de tratamento. Os sintomas de DTM, mesmo leves, promoveram impacto negativo sobre a qualidade de vida. Conclusão: Desta forma, os resultados apresentados sugerem necessidade de preparo e organização da rede de atenção à saúde, nos diferentes níveis, para garantir acesso e tratamento adequados aos pacientes com DTM e Dor Orofacial.

Palavras-chave: Transtornos da articulação temporomandibular; Prevalência; Atenção primária à saúde; Estresse psicológico; Qualidade de vida.

\footnotetext{
Abstract

Introduction: Epidemiological studies demonstrate a high prevalence of signs and/or symptoms of Temporomandibular Disorder (TMD) in the adult population. However, few studies have evaluated this dysfunction
} 
in primary healthcare. Aim: To assess the prevalence of TMD symptoms, associated factors and impact on quality of life in users of the primary healthcare network in the city of Maceió/AL. Method: 108 participants were evaluated for the presence of TMD symptoms (anamnestic index DMF), symptoms suggestive of anxiety and depression (Hospital Anxiety and Depression scale - HAD) and symptoms suggestive of stress (Adult Stress Symptoms Inventory - ISSL); in addition to quality of life related to oral health, assessed by the abridged version of the Oral Health-related (OHIP14). Results: $55.6 \%$ had symptoms of TMD and $19.4 \%$ showed a need for treatment. Female gender and self-report of parafunctional habits were predictors for TMD symptoms, and stress and habits, for treatment need. TMD symptoms, even mild, had a negative impact on quality of life. Conclusion: Thus, the results presented suggest the need for preparation and organization of the healthcare network, at different levels, to ensure adequate access and treatment for patients with TMD and Orofacial Pain.

Keywords: Temporomandibular joint disorders; Prevalence; Primary healthcare; Stress psychological; Quality of life.

\section{Resumen}

Introducción: Los estudios epidemiológicos demuestran una alta prevalencia de signos y/o síntomas del Trastorno Temporomandibular (TMD) em la población adulta. Sin embargo, pocos estudios han evaluado esta disfunción em la atención primaria de salud. Objetivo: Evaluar La prevalencia de síntomas de TMD, factores asociados e impacto em la calidad de vida em usuarios de la red de atención primaria de salud de la ciudad de Maceió/AL. Método: 108 participantes fueron evaluados por la presencia de síntomas de TMD (índice anamnésico DMF), síntomas sugestivos de ansiedad y depresión (escala Hospital Anxiety and Depression - HAD) y síntomas sugestivos de estrés (Inventario de Síntomas de Estrés para Adultos - ISSL); además de la calidad de vida relacionada con la salud bucal, evaluada por la versión abreviada del Oral Health-related (OHIP-14). Resultados: El 55,6\% tenía síntomas de TMD y el 19,4\% necesitaba tratamiento. El género femenino y el autoinforme de los hábitos parafuncionales fueron predictores de síntomas de TMD, y de estrés y hábitos para La necesidad de tratamiento. Los síntomas de TMD, incluso leves, tuvieron un impacto negativo em la calidad de vida. Conclusión: Así, los resultados presentados sugieren la necesidad de la preparación y organización de la red asistencial, en diferentes niveles, para asegurar un adecuado acceso y tratamiento a los pacientes con TMD y Dolor Orofacial.

Palabras clave: Trastornos de La articulación temporomandibular; Prevalencia; Atención primaria de salud; Estrés psicológico; Calidad de vida.

\section{Introdução}

As disfunções temporomandibulares (DTM) compreendem um grupo variado de sinais clínicos e sintomas que acometem a articulação temporomandibular (ATM), músculos mastigatórios e estruturas associadas ao aparelho estomatognático, como dentes e periodonto (De Leeuw \& Klasser, 2013). Sua etiologia é multifatorial, destacando-se diversos fatores que podem agir como predisponentes, perpetuantes ou desencadeantes (Chisnoiu et al., 2015; De Leeuw \& Klasser, 2013; Okeson, 2013). Entre estes fatores, podem ser mencionados: traumas e hipermobilidade articular (De Leeuw \& Klasser, 2013; Ögren et al., 2012); hábitos parafuncionais, incluindo bruxismo noturno e diurno (Paulino et al., 2018; Wagner \& Filho, 2018); fatores oclusais (Bilgiç \& Gelgör, 2017; Lemos, Moreira, et al., 2015); desordens do sono, como apnéia e ronco (Benoliel et al., 2017; Wagner \& Filho, 2018); fatores psicológicos, incluindo estresse, ansiedade e depressão (Fillingim et al., 2013; Yap \& Natu, 2020); diferenças hormonais (Guan et al., 2005; Hornung et al., 2020) e fatores genéticos, como o polimorfismos no transportador de serotonina (5HTT) e catecol-O-metiltransferase (COMT) (Brancher et al., 2019).

A prevalência de sinais e sintomas de DTM é elevada, variando de acordo com a população estudada e os instrumentos e/ou critérios para diagnóstico (De Leeuw \& Klasser, 2013), e estima-se, de forma conservadora, que a prevalência média de diagnósticos de DTM varie entre 10-15\% na população geral (List \& Jensen, 2017; Schiffman et al., 2014). Uma recente revisão sistemática com metanálise relacionada à DTM articular demonstrou uma prevalência de $31,1 \%$ de diagnósticos articulares em adultos e idosos, e uma prevalência de 11,3\% em crianças e adolescentes. Entre os diagnósticos específicos, o mais prevalente para ambos os grupos foi o deslocamento de disco com redução (Valesan et al., 2021). No Brasil, um estudo realizado com 1230 participantes mostrou que 39,2\% destes apresentavam sintomas de DTM e 25,6\% queixaram-se se dor relacionada a esta disfunção (Gonçalves et al., 2010). Além disso, diversos estudos têm demonstrado que a DTM ocorre mais frequentemente em adultos jovens, do sexo feminino e solteiros (Gonçalves et al., 2010; Moura et al., 2017; Paulino et al., 2018). 
Outros estudos têm demonstrado que os sintomas de DTM, especialmente relacionados à dor podem contribuir para a ocorrência de desordens psicológicas e comprometimento da qualidade de vida, principalmente em indivíduos com sinais clínicos articulares e musculares simultâneos (Lemos, Paulino, et al., 2015; Paulino et al., 2018; Tay et al., 2019). Os aspectos de maior comprometimento são a dor física, limitação funcional e desconforto psicológico (Lemos, Paulino, et al., 2015).

Desta forma, considerando a elevada prevalência e o impacto negativo sobre a qualidade de vida, a DTM apresenta uma grande relevância para a saúde pública (Melo et al., 2020; Michelotti et al., 2020; Oliveira et al., 2016; Schiffman et al., 2014). Contudo, poucos estudos avaliaram a prevalência desta disfunção, fatores associados e seu impacto nos diferentes níveis de atenção no Sistema Único de Saúde (SUS) brasileiro. A maioria avalia o perfil de pacientes atendidos em serviços especializados ofertados por instituições de ensino superior (Magri et al., 2018; Moura et al., 2017).

Sobre o tema, dois estudos realizados com usuários das Unidades Básicas de Saúde (UBSs) nas cidade Recife-PE (Mello et al., 2014) e Ponta Grossa-PR (Arruda; et al., 2012) demonstraram elevadas prevalências de DTM, 42\% e 67,2\% respectivamente.

Com relação à atenção secundária à saúde em Odontologia, em 2004 foi aprovada a Política Nacional de Saúde Bucal, visando ampliar o acesso aos serviços odontológicos. Como política relacionada à atenção secundária, foi incentivada a implantação dos Centros de Especialidades Odontológicas (CEOs). Estes são serviços de referência para a atenção primária que devem ofertar no mínimo as especialidades de Periodontia, Endodontia, Pacientes com Necessidades Especiais, Diagnóstico Bucal e Cirurgia Oral Menor (Figueiredo \& Goes, 2009). Contudo, a maioria dos CEOs oferta apenas as especialidades mínimas e não garantem serviços relacionados ao tratamento de pacientes com DTM e Dor Orofacial (Figueiredo \& Goes, 2009; Freitas et al., 2016).

Em face ao que foi apresentado, acredita-se que exista uma demanda reprimida de pacientes com sintomas de DTM e Dor Orofacial, que procuram inicialmente a atenção primária à saúde e podem não ter a garantia de acesso ao tratamento adequado neste nível ou na atenção secundária. Desta forma, este estudo objetiva avaliar a prevalência de DTM e fatores associados em usuários da atenção primária a saúde, visando a reformulação de estratégias e políticas para garantia da integralidade da atenção ao paciente com estas disfunções.

\section{Metodologia}

\subsection{Design do estudo}

Trata-se de um estudo transversal cuja amostra foi constituída por usuários da rede de atenção primária à saúde da cidade de Maceió - AL.

\subsection{Aspectos éticos}

A pesquisa foi submetida e aprovada pelo Comitê de Ética em Pesquisa da Universidade Federal de Alagoas (CEP/UFAL), CAAE: 30975620.7.0000.5013, e seguiu todas as determinações das resoluções 466/12, 510/2016 e 580/2018.

\subsection{Tamanho amostral}

Para determinação do tamanho amostral (n) adotou-se uma diferença mínima (p-P) de 10\%, nível de significância de 5\% ( $\left.\mathrm{z}_{\alpha / 2}=1,96\right)$ e proporção de casos de DTM (P) de 42\% (Mello et al., 2014), obtendo-se o $\mathrm{n}=94$. Com o acréscimo de um percentual de $10 \%$ de perdas, o n passou a ser de 104 participantes. Ao final do estudo, foram coletados os dados de 108 participantes, visando uma amostra adequada para realização da regressão logística binomial. 


\subsection{Critérios de elegibilidade}

O município de Maceió - AL apresenta 56 UBSs distribuídas em 08 distritos sanitários. Neste estudo, 36 UBSs foram selecionadas, mediante sorteio, com representação de todos os distritos. Os dados foram coletados no período entre setembro de 2020 e maio de 2021.

Foram incluídos os participantes com idade igual ou superior a 18 anos, sem distinção de gênero que se encontravam nas salas de espera das UBSs para atendimento odontológico, médico e enfermagem, que concordaram e assinaram o termo de Consentimento Livre e Esclarecido (TCLE).

Foram excluídos os participantes que relataram uso de anti-inflamatórios, analgésicos ou corticosteroides até três dias antes do estudo; com histórico de distúrbios neurológicos, neoplasias de cabeça e pescoço e doenças reumáticas.

\subsection{Instrumentos da pesquisa}

A prevalência de sintomas de DTM foi determinada por meio de um questionário de autopreenchimento contendo o índice anamnésico DMF. O índice é composto por 10 perguntas com as seguintes opções de resposta e pontuações: SIM (10), NÃO (0) ou ÀS VEZES (5). O somatório resulta na classificação dos participantes segundo a severidade da DTM: sem DTM (0 a 15 pontos); DTM leve (20 a 40); moderada (45 a 65) e severa (70 a 100). O DMF também classifica os participantes em indivíduos sem necessidade de tratamento (Sem DTM e DTM leve) e com necessidade de tratamento (DTM moderada e severa) (Lemos, Moreira, et al., 2015; Lemos, Paulino, et al., 2015; Paulino et al., 2018).

Para avaliação da presença de sintomas sugestivos de ansiedade e depressão foi utilizada a escala Hospital Anxiety and Depression - HAD (Paulino et al., 2018). A escala possui 14 itens, sendo sete voltados para a avaliação da ansiedade (HAD-A) e sete para a depressão (HAD-D). Cada um dos itens pode ser pontuado de 0-3, com uma pontuação máxima de 21 pontos por subescala. A pontuação obtida permite a seguinte classificação dos participantes: Sem sintomas de ansiedade (0-8) e com sintomas ( $\geq 9$ ) (HAD-A); sem sintomas de depressão (0-8) e com sintomas ( $\geq 9$ ) (HAD- D).

A presença de sintomas sugestivos de estresse foi determinada através do Inventário de Sintomas de Stress para Adultos - ISSL (Lipp, 2005). O instrumento permite identificar sintomas de estresse e suas fases (alerta, resistência, quaseexaustão e exaustão). O inventário é composto por 53 questões divididas em três quadros que incluem as quatro fases do estresse. Os quadros referem-se a sintomas apresentados, respectivamente, nas últimas 24 horas, última semana e o último mês. São 34 itens referem-se aos sintomas físicos e 19 aos psicológicos. A presença de sintomas de estresse é determinada pelo registro positivo de 8 ou mais perguntas para o quadro 1 (fase de alerta), 4 ou mais para o quadro 2 (fase de resistência e quase exaustão) e 9 ou mais para o quadro 3 (fase de exaustão).

A qualidade de vida relacionada com a saúde oral foi determinada por meio da versão resumida do Oral Healthrelated - OHIP-14 (Lemos, Paulino, et al., 2015; Paulino et al., 2018). O questionário é composto por 14 perguntas com as possibilidades de respostas "nunca", "raramente", "às vezes", "repetidamente" e "sempre", com os respectivos valores de zero, um, dois, três e quatro. O somatório de todas as questões produz a pontuação total do OHIP-14, que pode variar de zero a 56, com pontuações mais altas, significando impacto negativo na saúde.

O OHIP-14 também é dividido em 7 domínios, sendo dois itens para cada domínio: limitação funcional (itens 1 e 2), dor física (itens 3 e 4), desconforto psicológico (itens 5 e 6), incapacidade física (Itens 7 e 8), incapacidade psicológica (itens 9 e 10), incapacidade social (itens 11 e 12), e deficiência (itens 13 e 14). A pontuação de cada um dos sete domínios do OHIP-14 varia de zero a oito pontos, com maiores escores significando impacto mais negativo (Lemos, Paulino, et al., 2015).

\subsection{Análises estatísticas}

Os dados foram tabulados no Software estatístico gratuito Jamovi®, versão 1.6 (The jamovi Project, 2021), e 
apresentados na forma de estatística descritiva (frequência relativa e absoluta) e inferencial. A influência dos preditores sexo, autorrelato de hábitos parafuncionais, sintomas de ansiedade, depressão e estresse, sobre a presença de DTM e necessidade de tratamento foi determinada em um modelo de regressão logística múltipla binomial.

Para determinação do impacto dos sintomas e severidade da DTM sobre a qualidade de vida relacionada com a saúde oral foi realizado inicialmente o teste de normalidade Shapiro-Wilk, o qual demonstrou que os dados não apresentavam distribuição normal. Desta forma, as comparações entre os grupos presença/ausência de DTM ou necessidade de tratamento foi realizada por meio do teste de Mann-Whitney U. As comparações relacionados a severidade de DTM foram determinadas por meio do teste de Kruskal-Wallis, seguido do teste de Dwass-Steel-Critchlow-Fligner para comparações múltiplas.Em todas as análises foi adotado um nível de significância de $5 \%(\mathrm{p}<0,05)$.

\section{Resultados}

Neste estudo, foram avaliados os dados de 108 participantes. A maioria era do sexo feminino ( $\mathrm{n}=63,58,3 \%)$ e a idade média foi de 37,08 anos (DP 14,74). Conforme se observa na Tabela 1, uma parcela considerável dos participantes apresentou sintomas de DTM (55,6\%). Contudo, a maioria exibiu sintomas leves e apenas 19,4\% apresentaram necessidade de tratamento.

Tabela 1 - Prevalência de sintomas de DTM (Índice DMF) em usuários da rede de atenção primária à saúde, Maceió-AL, 2021.

\begin{tabular}{|cll|}
\hline Presença de DTM & $\mathbf{n}$ & $\%$ \\
\hline Sem DTM & 48 & 44.4 \\
Com DTM & 60 & 55,6 \\
\hline Necessidade de tratamento & $\mathbf{n}$ & $\mathbf{\%}$ \\
\hline Sem Necessidade & & \\
Com Necessidade & 87 & 80.6 \\
Severidade da DTM & 21 & 19.4 \\
\hline Sem DTM & $\mathbf{n}$ & $\mathbf{\%}$ \\
DTM Leve & 48 & 44.4 \\
DTM Moderada & 39 & 36.1 \\
DTM Severa & 18 & 16.7 \\
\hline
\end{tabular}

Fonte: Autores do estudo (2021).

Em relação aos possíveis preditores, 43,5\% relataram presença de hábitos parafuncionais (Tabela 2). As frequências dos hábitos relatados foram: bruxismo possível $(16,34 \%)$, colocar a mão no queixo $(11,94 \%)$, dormir de lado $(11,32 \%)$, roer unhas $(10,06 \%)$, mastigação unilateral $(9,43 \%)$, morder objetos $(8,17 \%)$, morder a bochecha $(8,17 \%)$, mascar chiclete $(8,17 \%)$, morder a língua $(7,54 \%)$, morder o lábio $(7,54 \%)$ e mastigar gelo $(1,25 \%)$.

A maioria dos participantes não apresentou sintomas sugestivos de ansiedade, depressão e estresse. Entre aqueles com sintomas de estresse, a maioria encontrava-se na fase de resistência (Tabela 2). 
Tabela 2 - Frequência de hábitos parafuncionais (autorrelato), sintomas de ansiedade e depressão (Escala Hospital Anxiety and Depression - HAD) e estresse (Inventário de Sintomas de Stress para Adultos - ISSL) em usuários da rede de atenção primária à saúde, Maceió-AL, 2021.

\begin{tabular}{|lll|}
\hline Hábitos parafuncionais & $\mathbf{n}$ & $\mathbf{\%}$ \\
\hline Sem Hábitos & 61 & 56.5 \\
Com Hábitos & 47 & 43.5 \\
\hline Sintomas de Ansiedade & $\mathbf{n}$ & $\mathbf{\%}$ \\
\hline Sem Ansiedade & 79 & 73.1 \\
Com Ansiedade & 29 & 26.9 \\
\hline Sintomas de Depressão & $\mathbf{n}$ & $\mathbf{\%}$ \\
\hline Com Depressão & 93 & 86.1 \\
Sem Depressão & 15 & 13.9 \\
\hline Sintomas de estresse & $\mathbf{n}$ & $\mathbf{\%}$ \\
\hline Sem estresse & 65 & 60.2 \\
Com estresse & 43 & 39.8 \\
\hline Classificação dos sintomas de estresse & $\mathbf{n}$ & $\mathbf{\%}$ \\
\hline Fase de alerta & 1 & 2.3 \\
Fase de resistência & 34 & 79.1 \\
Fase de quase exaustão & 4 & 9.3 \\
Fase de exaustão & 4 & 9.3 \\
\hline
\end{tabular}

Fonte: Autores do estudo (2021)

Os dados referentes à influência dos preditores sexo, autorrelato de hábitos parafuncionais, sintomas de estresse, ansiedade e depressão na presença e necessidade de tratamento para DTM foram apresentados na Tabela 3. Para ambos os modelos de regressão, os valores de VIF e Tolerância indicaram ausência de multicolinearidade (dados não apresentados). A ausência de multicolinearidade é um pré-requisito para a realização de uma regressão logística.

Em relação à presença de sintomas de DTM, o modelo de regressão logística múltipla binomial contendo todos as variáveis previsoras foi significativo: $\left[\mathrm{X}^{2}(5)=49.1 ; \mathrm{P}<0.001 ; \mathrm{R}^{2} \mathrm{Negelkerke}=0.4890\right]$.

Neste modelo, o sexo feminino $(\mathrm{OR}=3.294 ; \mathrm{RP}=1.66 ;$ IC $95 \%=1.0443-10.391)$ e o autorrelato de hábitos parafuncionais $(\mathrm{OR}=16.771 ; \mathrm{RP}=2.4 ; \mathrm{IC} 95 \%=5.1678-54.425)$ foram preditores significativos para a presença de sintomas de DTM.

O modelo de regressão logística com inclusão de todas as variáveis preditoras para a necessidade de tratamento também foi significativo: $\left[\mathrm{X}^{2}(5)=38.8 ; \mathrm{P}<0.001 ; \mathrm{R}^{2}\right.$ Negelkerke $\left.=0.481\right]$.

Para a necessidade de tratamento, apenas o autorrelato de hábitos parafuncionais $(\mathrm{OR}=18.18382 ; \mathrm{RP}=7.79 ; \mathrm{IC} 95 \%$ $=3.60130-91.8145)$ e presença de sintomas de estresse $(\mathrm{OR}=7.08369 ; \mathrm{RP}=6.42 ; \mathrm{IC} 95 \%=1.76524-28.426)$ foram preditores significativos. 
Tabela 3 - Regressão logística múltipla binomial para verificar se as variáveis sexo, autorrelato de hábitos parafuncionais, sintomas de ansiedade, depressão e estresse são preditors para a presença de sintomas de DTM e necessidade de tratamento, Maceió-AL, 2021.

\begin{tabular}{|c|c|c|c|c|c|c|c|}
\hline $\begin{array}{l}\text { Preditores para presença de Sintomas } \\
\text { de DTM }\end{array}$ & $\bar{B}$ & SE & $\mathbf{Z}$ & p & OR & $\mathbf{R P}$ & IC 95\% \\
\hline Intercept & -2.229 & 0.552 & -4.039 & $<0.001$ & 0.108 & & $0.0365-0.318$ \\
\hline $\begin{array}{l}\text { Sexo } \\
\qquad \text { (Feminino - Masculino) }\end{array}$ & 1.192 & 0.586 & 2.034 & 0.042 & 3.294 & 1.66 & $1.0443-10.391$ \\
\hline $\begin{array}{l}\text { Hábitos } \\
\text { (Com Hábitos - Sem Hábitos) }\end{array}$ & 2.820 & 0.601 & 4.695 & $<0.001$ & 16.771 & 2.4 & $5.1678-54.425$ \\
\hline $\begin{array}{l}\text { Ansiedade } \\
\quad \text { (Com ansiedade - Sem ansiedade) }\end{array}$ & 1.064 & 0.671 & 1.587 & 0.113 & 2.899 & 1.69 & $0.7784-10.798$ \\
\hline $\begin{array}{l}\text { Depressão } \\
\quad \text { (Com Depressão - Sem Depressão) }\end{array}$ & 0.607 & 0.749 & 0.810 & 0.418 & 1.834 & 1.24 & $0.4227-7.958$ \\
\hline $\begin{array}{l}\text { Estresse } \\
\qquad(\text { Com estresse }- \text { Sem estresse })\end{array}$ & 0.938 & 0.556 & 1.686 & 0.092 & 2.554 & 1.84 & $\begin{array}{ll}0.8586 & -7.597\end{array}$ \\
\hline $\begin{array}{l}\text { Preditores para Necessidade de } \\
\text { Tratamento }\end{array}$ & B & SE & $\mathbf{Z}$ & $\mathbf{p}$ & OR & & IC 95\% \\
\hline Intercept & -4.784 & 0.976 & -4.902 & $<0.001$ & 0.00836 & & $0.00123-0.0566$ \\
\hline $\begin{array}{l}\text { Sexo } \\
\text { (Feminino - Masculino) }\end{array}$ & 0.117 & 0.761 & 0.154 & 0.878 & 1.12408 & 1.76 & $0.25286-4.9971$ \\
\hline $\begin{array}{l}\text { Hábitos } \\
\text { (Com Hábitos - Sem Hábitos) }\end{array}$ & 2.901 & 0.826 & 3.511 & $<0.001$ & 18.18382 & 7.79 & $3.60130-91.8145$ \\
\hline $\begin{array}{l}\text { Ansiedade } \\
\quad(\text { Com ansiedade }- \text { Sem ansiedade })\end{array}$ & 0.447 & 0.766 & 0.584 & 0.559 & 1.56393 & 2.47 & $0.34834-7.0215$ \\
\hline $\begin{array}{l}\text { Depressão } \\
\quad \text { (Com Depressão - Sem Depressão) }\end{array}$ & 1.385 & 0.984 & 1.408 & 0.159 & 3.99550 & 1.93 & $0.58112-27.4714$ \\
\hline $\begin{array}{l}\text { Estresse } \\
\qquad \text { (Com estresse }- \text { Sem estresse })\end{array}$ & 1.958 & 0.709 & 2.762 & 0.006 & 7.08369 & 6.422 & $1.76524-28.426$ \\
\hline
\end{tabular}

B=Coeficiente da equação; SE = Erro Padrão; $Z$ = Estatística de Wald; OR = OddsRatio; RP = Razão de Prevalência. Fonte: Autores do estudo (2021).

Em relação à qualidade de vida, participantes com sintomas de DTM exibiram pontuações do OHIP-14 estatisticamente maiores do que participantes sem sintomas de DTM, indicando um impacto negativo sobre a qualidade de vida relacionada à saúde oral. Resultado semelhante foi observado para o grupo necessidade de tratamento (Tabela 4).

Tabela 4 - Diferenças entre os valores do OHIP-14 nos grupos presença, necessidade de tratamento e severidade da DTM, Maceió-AL, 2021.

\begin{tabular}{lccc}
\hline & Mediana & $\begin{array}{c}\text { Intervalo } \\
\text { interquartílico }\end{array}$ & p \\
\hline Sem DTM & 4 & $0.75-10$ & $<0.001^{*}$ \\
Com DTM & 13 & $7-20$ & \\
& & & $2-15.5$ \\
Sem necessidade & 8 & $12-22$ & $<0.001^{*}$ \\
Com necessidade & 15 & & \\
Sem DTM & 4 & $0.75-10$ & $0.005^{* *}$ \\
DTM Leve & 13 & $4.5-19$ & $<0.001^{* *}$ \\
DTM Moderada & 14 & $10.5-21.3$ & 0.057 \\
DTM Severa & 21 & $17-28$ & \\
\hline
\end{tabular}

* Diferença estatística significativa. Teste de Mann-Whitney U

** Diferença estatística significativa. Teste de Dwass-Steel-Critchlow-Fligner

Fonte: Autores do estudo (2021).

O Teste de Kruskal-Wallis mostrou diferença estatisticamente significante entre as categorias de severidade da DTM $(\mathrm{P}<0,001)$. Já o teste de Dwass-Steel-Critchlow-Fligner foi aplicado para determinação de quais grupos eram diferentes. Observou-se que os participantes com sintomas de DTM leve e moderada exibiram valores estatisticamente maiores do OHIP- 
14 em comparação aos participantes sem sintomas de DTM. Os participantes com DTM severa exibiram maiores pontuações do OHIP-14 em comparação aos participantes sem sintomas de DTM. Contudo, como a amostra de participantes com DTM severa foi pequena, não foi possível demonstrar uma diferença estatística significativa (Tabela 4).

Os domínios do OHIP-14 mais afetados nos participantes com presença de sintomas de DTM foram: limitação funcional (OHIP1), dor física (OHIP2), desconforto psicológico (OHIP3), incapacidade física (OHIP4), incapacidade psicológica (OHIP5) e incapacidade Social (OHIP6). Nos participantes com necessidade de tratamento, apenas os quatro primeiros (OHIP-1 - OHIP4) mostram diferenças significativas em comparação os participantes em necessidade de tratamento (Tabela 5).

Tabela 5 - Diferenças entre os valores de cada domínio do OHIP-14 nos grupos presença e necessidade de tratamento da DTM, Maceió-AL, 2021

\begin{tabular}{|c|c|c|c|c|}
\hline Domínios do OHIP-14 & Presença de DTM & Mediana & Intervalo interquartílico & $\mathbf{p}$ \\
\hline \multirow{2}{*}{ OHIP1: Limitação Funcional } & Ausência & 0 & $0-1$ & \multirow{2}{*}{$<0.001 *$} \\
\hline & Presença & 1 & $0-2$ & \\
\hline \multirow{2}{*}{ OHIP2: Dor Física } & Ausência & 0 & $0-2$ & \multirow{2}{*}{$<0.001 *$} \\
\hline & Presença & 3 & $1.75-4$ & \\
\hline \multirow{2}{*}{$\begin{array}{l}\text { OHIP3: Desconforto } \\
\text { Psicológico }\end{array}$} & Ausência & 1 & $0-3$ & \multirow{2}{*}{$<0.001 *$} \\
\hline & Presença & 4 & $2-5$ & \\
\hline \multirow{2}{*}{ OHIP4: Incapacidade Física } & Ausência & 0 & $0-0$ & \multirow{2}{*}{$<0.001 *$} \\
\hline & Presença & 1 & $0-2$ & \\
\hline \multirow{2}{*}{$\begin{array}{l}\text { OHIP5: Incapacidade } \\
\text { Psicológica }\end{array}$} & Ausência & 0 & $0-2$ & \multirow{2}{*}{$0.001 *$} \\
\hline & Presença & 2 & $0-4$ & \\
\hline \multirow{2}{*}{ OHIP6: Incapacidade Social } & Ausência & 0 & $0-2$ & \multirow{2}{*}{$0.042 *$} \\
\hline & Presença & 1 & $0-3.25$ & \\
\hline \multirow{2}{*}{ OHIP7: Deficiência } & Ausência & 0 & $0-0,25$ & \multirow{2}{*}{0.112} \\
\hline & Presença & 0 & $0-1$ & \\
\hline OHIP-14 & $\begin{array}{l}\text { Necessidade de } \\
\text { tratamento }\end{array}$ & Mediana & Intervalo interquartílico & $\mathbf{p}$ \\
\hline \multirow{2}{*}{ OHIP 1: Limitação Funcional } & Ausência & 0 & $0-1.5$ & \multirow{2}{*}{$<0.001 *$} \\
\hline & Presença & 2 & $1-3$ & \\
\hline \multirow{2}{*}{ OHIP 2: Dor Física } & Ausência & 1 & $0-2.5$ & \multirow{2}{*}{$<0.001 *$} \\
\hline & Presença & 4 & $3-6$ & \\
\hline \multirow{2}{*}{$\begin{array}{l}\text { OHIP 3: Desconforto } \\
\text { Psicológico }\end{array}$} & Ausência & 2 & $0-4$ & \multirow{2}{*}{$<0.001 *$} \\
\hline & Presença & 4 & $3-5$ & \\
\hline \multirow{2}{*}{ OHIP 4: Incapacidade Física } & Ausência & 0 & $0-1$ & \multirow{2}{*}{$0.003 *$} \\
\hline & Presença & 1 & $0-3$ & \\
\hline \multirow{2}{*}{$\begin{array}{l}\text { OHIP 5: Incapacidade } \\
\text { Psicológica }\end{array}$} & Ausência & 1 & $0-2$ & \multirow{2}{*}{0.085} \\
\hline & Presença & 2 & $1-3$ & \\
\hline \multirow{2}{*}{ OHIP 6: Incapacidade Social } & Ausência & 0 & $0-3$ & \multirow{2}{*}{0.963} \\
\hline & Presença & 1 & $0-2$ & \\
\hline \multirow[t]{2}{*}{ OHIP 7: Deficiência } & Ausência & 0 & $0-1$ & \multirow{2}{*}{0.22} \\
\hline & Presença & 0 & $0-2$ & \\
\hline
\end{tabular}

*Diferença estatística significativa. Teste Mann-Whitney U.

Fonte: Autores do estudo (2021).

\section{Discussão}

Neste estudo, utilizou-se o índice Anamnésico DMF para determinação dos sintomas de DTM na amostra avaliada. O índice DMF tem as vantagens de ser um instrumento simples, fácil e de baixo custo, o que favorece seu uso em estudos epidemiológicos populacionais (Chaves et al., 2008a) ou em metodologias sem a presença física do participante, como em pesquisas por meio de telefone, correios ou internet (Campos et al., 2014). Além disso, sua habilidade de diferenciar pacientes com e sem DTM é considerada excelente, razão pela qual sua adaptação tem sido realizada para diversos outros idiomas (Sánchez-Torrelo et al., 2020). Contudo, limita-se a determinação da severidade dos sintomas de DTM, não permitindo uma classificação diagnóstica. 
Atualmente os instrumentos mais amplamente utilizados e aceitos para uma classificação diagnostica da DTM são o Research Diagnostic Criteria for Temporomandibular Disorders (RDC/TMD) (Chaves et al., 2008b) e mais recentemente, o Diagnostic Criteria for Temporomandibular Disorders (DC/TMD) (Pereira-Junior \& Gonçalves, 2020; Schiffman et al., 2014). Este último é subdividido em quatro instrumentos: um questionário de triagem da dor para DTM, questionário de sintomas, questionário de dados demográficos e um protocolo de exame físico para dor (Pereira-Junior \& Gonçalves, 2020; Schiffman et al., 2014). Os dois instrumentos, apesar de aceitos internacionalmente, são longos e exigem um treinamento adequado da equipe, dificultando suas aplicações em estudos populacionais (Paulino et al., 2018) ou em exames de rotina na atenção primária a saúde.

Além disso, um estudo demonstrou que o índice DMF apresentou alto grau de precisão para o diagnóstico de DTM miogênica (área sob a curva ROC de 0,94), com melhor ponto de corte sendo a pontuação de 47,5 (Berni et al., 2015). A versão resumida do índice DMF (exclusão das perguntas 4,5, 8, 9, 10) também apresentou elevada acurácia para DTM miogênica (área sob a curva ROC de 0,97) e melhor ponto de corte 17,5 (Pires et al., 2018). Em ambos os estudos, o instrumento RDC/TMD foi utilizado como padrão-ouro.

No presente estudo, observou-se que a prevalência de DTM por meio do instrumento DMF foi elevada (55,6\%). A maioria dos participantes apresentou sintomas leves (36,1\%) e 19,4\% apresentaram necessidade de tratamento. Estes dados são semelhantes aos descritos em estudos prévios que utilizaram o mesmo instrumento em populações de não pacientes (Bonjardim et al., 2009; Medeiros et al., 2011; Oliveira et al., 2006; Paulino et al., 2018).

No presente estudo, a amostra consistiu em participantes que estavam nas salas de espera das UBSs da cidade de Maceió-AL para atendimento médico, odontológico ou pela enfermagem. Um estudo realizado na cidade de Ponta Grossa-PR com uma amostra semelhante e mesmo instrumento demonstrou uma prevalência de sintomas de DTM maior (67,2\%) (Arruda; et al., 2012). Por outro lado, um estudo realizado na cidade de Recife-PE, com uma amostra semelhante, porém utilizando o instrumento RDC/TMD, demonstrou um percentual menor de participantes com o diagnóstico de DTM (42\%) (Mello et al., 2014).Outro estudo realizado na cidade de Maringá-PR, utilizando o RDC/TMD mostrou que 29,5\% dos indivíduos avaliados (usuários de UBSs) apresentavam distúrbios musculares, 7,9\%, deslocamento de disco e 39,1\%, outros distúrbios articulares (Progiante et al., 2015).

Estes resultados e aqueles descritos em estudos prévios (Arruda; et al., 2012; Mello et al., 2014; Progiante et al., 2015)sugerem a existência de uma demanda considerável de indivíduos com sintomas de DTM e Dor Orofacial na atenção primária à saúde. Estes potenciais pacientes precisam ser acolhidos pelo Sistema Único de Saúde (SUS), avaliados e, quando necessário, encaminhados para serviços especializados, permitindo um diagnóstico e tratamento adequados (Melo et al., 2020).

Contudo, a assistência a estes pacientes apresenta algumas barreiras, principalmente relacionadas ao preparo das equipes e disponibilidade de serviços, seja na atenção primária ou secundária. Na escassez de serviços no SUS para tratamento da DTM e Dor Orofacial, estes pacientes acabam tendo como opção mais acessível os serviços ofertados por Faculdades de Odontologia ou Ambulatórios vinculados as Instituições de ensino Superior (Melo et al., 2020; Magri et al., 2018; Moura et al., 2017), que se localizam quase sempre nas grandes regiões metropolitanas.

Com relação ao preparo das equipes, um estudo realizado no Estado da Paraíba demonstrou baixa concordância de cirurgiões-dentistas clínicos gerais com especialistas em DTM e Dor Orofacial em relação aos aspectos etiológicos desta disfunção (Assis et al., 2015). Outro estudo realizado com alunos do último ano da graduação em Odontologia mostrou que o conhecimento dos alunos sobre os conceitos básicos relacionados à DTM era limitado, e a maioria não tinha segurança para tratar pacientes com esta disfunção (Tormes et al., 2020). Salientando essa necessidade, uma publicação recente enfatiza que o ensino da Dor Orofacial, que inclui dentro de suas competências as Disfunções Temporomandibulares, em todos os níveis 
educacionais (universitário, pós-graduação e educação continuada) é essencial para melhorar o atendimento odontológico e a qualidade de vida dos pacientes que sofrem dessas condições (Costa et al., 2021).

Estudos de base populacional, com instrumentos padronizados, são necessários para traçar um panorama da epidemiologia da DTM no Brasil, bem como trabalhos que avaliem a oferta de serviços relacionados a esta especialidade (Melo et al., 2020). Neste sentido, nossa equipe está desenvolvendo um estudo nacional, em fase de coleta de dados, para avaliar a oferta de serviços para DTM e Dor Orofacial, e o nível de conhecimento e condutas clínicas de cirurgiões-dentistas que atuam no SUS.

Em relação aos possíveis preditores, a análise por meio de regressão logística múltipla binomial mostrou que as variáveis sexo feminino e autorrelato de hábitos parafuncionais são importantes preditores para a presença de sintomas de DTM. Enquanto as variáveis autorrelato de hábitos parafuncionais e sintomas de estresse foram importantes preditores para a necessidade e tratamento, que indica a presença de sintomas mais severos.

Corroborando com os resultados apresentados, estudos prévios também demonstraram uma associação positiva entre o sexo feminino e a presença de sintomas ou sinais clínicos de DTM (Gonçalves et al., 2010; Moura et al., 2017; Paulino et al., 2018). Uma recente metanálise mostrou que mulheres apresentam maior prevalência de DTM em todos os grupos diagnósticos do RDC/TMD. Demonstrou-se também que mulheres apresentam o risco duas vezes maior para desenvolverem DTM em comparação aos homens, na avaliação de estudos de base populacional (Bueno et al., 2018). Contudo, ainda não está claro as bases biológicas e biopsicossociais desta relação.

Acredita-se que a maior prevalência de DTM em mulheres deve-se a maior preocupação destas com a saúde, refletindo em maior procura por serviços relacionados à DTM e Dor Orofacial(Moura et al., 2017; Paulino et al., 2018). Por outro lado, estudos realizados em modelos animais de inflamação têm demonstrado que a redução dos níveis de estrógeno resultou em redução da inflamação da articulação temporomandibular (Guan et al., 2005)e do comportamento nociceptivo (Hornung et al., 2020), sugerindo que o estrógeno pode estar associado à fisiopatologia desta disfunção (List \& Jensen, 2017).

Consistente com os resultados apresentados, outros estudos também têm demonstrado uma associação positiva entre hábitos orais parafuncionais, especialmente o bruxismo, e a presença de DTM (Paduano et al., 2020; Paulino et al., 2018). Acredita-se que a atividade parafuncional possa causar instabilidade do sistema oclusal, devido à sobrecarga oclusal e presença de forças direcionadas horizontalmente (Manfredini et al., 2003). Além disso, a contração isométrica dos músculos mastigatórios está associada a eventos musculares isquêmicos, podendo resultar em espasmos musculares, fadiga e dor (Manfredini et al., 2003; Okeson, 2013).

Sobre o tema, uma recente metanálise mostrou que crianças com bruxismo têm 2,97 vezes mais chance de apresentar DTM (Reis et al., 2019). Já uma revisão sistemática demonstrou uma forte relação entre o bruxismo e DTM em indivíduos adultos (Jimenez-Silva et al., 2017). Contudo, a força de evidência para ambos os estudos foi considerada baixa, devido ao elevado risco de viés nos estudos primários incluídos nas revisões.

Um dos principais motivos para as controvérsias ou inconsistências nos estudos é a falta de clareza quanto ao diagnóstico adequado do bruxismo. Atualmente o bruxismo é definido como uma atividade muscular mastigatória durante o sono (bruxismo do sono) ou vigília (bruxismo em vigília) que pode ter um efeito protetor para determinados distúrbios, como a apneia, ou representar um fator de risco para a DTM (Lobbezoo et al., 2013, 2018). Baseado nos diferentes instrumentos, o bruxismo pode ser classificado em possível, quando determinado pelo autorrelato do paciente e/ou familiares próximos; provável, determinado por meio de avaliação clínica intraoral, com ou sem o autorrelato; e definitivo, através de avaliação por meio de eletromiografia (bruxismo em vigília) e polissonografia (bruxismo noturno), com ou sem o autorrelato e avaliação intraoral (Lobbezoo et al., 2018). 
Neste estudo, a presença de hábitos parafuncionais foi determinada pelo autorrelato do participante, contudo, não foi utilizada a classificação de atividade do sono ou em vigília. Entre os hábitos mais prevalentes na amostra avaliada, destacou-se o bruxismo possível (16.34\%). Desta forma, mais estudos com instrumentos precisos como a eletromiografia e polissonografia são necessários para avaliar a relação entre a presença de sintomas de DTM e o bruxismo definitivo na população avaliada.

Atualmente o modelo biopsicossocial tem destacado a importância dos fatores psicológicos na etiologia da DTM e hábitos parafuncionais, como o bruxismo (De Leeuw \& Klasser, 2013; Manfredini et al., 2003; Okeson, 2013). Neste estudo, demonstrou-se que a presença de sintomas de estresse foi relacionada à necessidade de tratamento. De acordo com o índice DMF, a necessidade de tratamento representa a presença de sintomas mais severos (sintomas moderados e graves). Desta forma, os resultados sugerem que o estresse pode ser um importante fator perpetuante para a DTM, e envolvido em uma maior gravidade dos sinais e sintomas.

Consistente com estes resultados, estudos prévios também mostraram associação positiva entre a presença de estresse emocional e sintomas/sinais de DTM (Augusto et al., 2016; Yap \& Natu, 2020). Além disso, um estudo de coorte que avaliou prospectivamente participantes por um período médio de 2,8 anos demonstrou que diversas variáveis psicológicas, incluindo o estresse emocional, aumentaram o risco de desenvolvimento da DTM (Fillingim et al., 2013).

Também tem sido demonstrado na literatura que os sintomas de DTM além de suas íntimas relações com os fatores psicossociais, impactam negativamente na qualidade de vida (Lemos, Paulino, et al., 2015; Paulino et al., 2018; Tay et al., 2019). Neste estudo, observou-se que a presença de sintomas de DTM, mesmo os sintomas leves, e a necessidade de tratamento resultaram em impacto negativo sobre a qualidade de vida relacionada com a saúde oral em comparação aos indivíduos sem sintomas de DTM. Corroborando com estes resultados, uma recente metanálise demonstrou que todos os subgrupos de diagnóstico da DTM determinados pelo RDC/TMD apresentaram impacto negativo sobre a qualidade de vida, especialmente os grupos I (desordens musculares) e III (artralgia/artrite/artrose) (Pigozzi et al., 2021).

No conjunto, os resultados do presente estudo indicam uma necessidade de avaliação destes potenciais pacientes, pois os sintomas de DTM, mesmo leves, foram capazes de impactar negativamente na qualidade de vida. Conforme discutido no início deste tópico, a atenção adequada a estes pacientes passa também pela necessidade de preparo e organização da rede de assistência à saúde, nos diferentes níveis de atenção. Esse processo inclui uma série de "nós-críticos" que podem e devem ser considerados nesse processo. Um exemplo é a ausência de dados populacionais a respeito da prevalência da DTM e outras dores orofaciais na população brasileira, que poderia ser solucionado pela inclusão de índices de DTM, ou de outras patologias não dentárias que têm como sintoma a dor orofacial, no "Levantamento das condições de saúde bucal da população brasileira" realizado pelo Ministério da Saúde, o que daria informações valiosas no planejamento do serviço (Melo et al., 2020). Melhorar a formação universitária dos cirurgiões-dentistas a respeito desse tema também é a uma forma de garantir que profissionais bem formados promovam um bom atendimento dentro dos níveis de complexidade e entendendo e agilizando o sistema de referência e contra referência de pacientes no SUS (Melo et al., 2020), evitando diagnósticos tardios e tratamentos ineficazes que podem piorar o quadro de saúde dos pacientes e gerar problemas mais sérios como cronificação da dor, por exemplo (Costa et al., 2021).

Além disso, a melhoria das políticas públicas que promovam acesso da população ao atendimento adequado, tanto dentro da atenção primária, quanto pela possível inclusão de especialistas em Disfunção Temporomandibular e Dor Orofacial, nos CEOs, tem o papel de ampliar e melhorar o acesso ao atendimento odontológico integral a uma parcela maior da população que sofre com os sinais e sintomas dessas alterações, prevenindo complicações mais graves e melhorando sua qualidade de vida (Melo et al., 2020; Oliveira et al., 2016; Paulino et al., 2018). 


\section{Conclusão}

A prevalência de sintomas de DTM foi elevada na amostra de usuários da rede de atenção primária a saúde. A maioria apresentou sintoma leves e 19,4\% apresentaram necessidade de tratamento. Contudo, os sintomas de DTM, mesmo os leves, promoveram um impacto negativo sobre a qualidade de vida relacionada com a saúde oral. Além disso, o sexo feminino e o autorrelato de hábitos parafuncionais foram importantes preditores para a presença de sintomas de DTM. O autorrelato de hábitos parafuncionais e o estresse foram preditores para a necessidade de tratamento.

Considerando as limitações do estudo, os resultados sugerem uma grande demanda de pacientes com sintomas de DTM na atenção primária do SUS. Investimento em capacitação da equipes de saúde bucal e reformulação da rede são necessários, para receber estes pacientes, realizar o tratamento inicial, e encaminhá-los para outros níveis de atenção ou especialidades, quando necessário.

\section{Referências}

Arruda, E. P., Assad, R. A., Gabardo, M. L., Matei, G. C., \& Almeida, B. C. (2012). Prevalência da disfunção temporomandibular em pacientes das unidades básicas de saúde de Ponta Grossa-PR. Revista Ortodontia Gaúcha, 16(2), 5-11.

Assis, J. F. C., Silva, P. L. P., Lima, J. A. S., Forte, F. D. S., \& Batista, A. U. D. (2015). The knowledge level of dental surgeons regarding the relationship between occlusal factors and Temporomandibular Disorders (TMD). Revista de Odontologia Da UNESP, 44(6), 360-367. https://doi.org/10.1590/18072577.11615 .

Augusto, V. G., Bueno Perina, K. C., Gontijo Penha, D. S., Santos, D. C. A., \& Oliveira, V. A. S. (2016). Temporomandibular dysfunction, stress and common mental disorder in university students. Acta Ortopedica Brasileira, 24(6), 330-333. https://doi.org/10.1590/1413-785220162406162873.

Benoliel, R., Zini, A., Zakuto, A., Slutzky, H., Haviv, Y., Sharav, Y., \& Almoznino, G. (2017). Subjective Sleep Quality in Temporomandibular Disorder Patients and Association with Disease Characteristics and Oral Health-Related Quality of Life. Journal of Oral \& Facial Pain and Headache, 31(4), 313-322. https://doi.org/10.11607/ofph.1824.

Berni, K. C. S., Dibai-Filho, A. V., \& Rodrigues-Bigaton, D. (2015). Accuracy of the Fonseca anamnestic index in the identification of myogenous temporomandibular disorder in female community cases. Journal of Bodywork and Movement Therapies, 19(3), 404-409. https://doi.org/10.1016/j.jbmt.2014.08.001.

Bilgiç, F., \& Gelgör, I. E. (2017). Prevalence of temporomandibular dysfunction and its association with malocclusion in children: An epidemiologic study. Journal of Clinical Pediatric Dentistry, 41(2), 161-165. https://doi.org/10.17796/1053-4628-41.2.161.

Bonjardim, L. R., Lopes-Filho, R. J., Amado, G., Albuquerque, R. L. C., \& Goncalves, S. R. J. (2009). Association between symptoms of temporomandibular disorders and gender, morphological occlusion, and psychological factors in a group of university students. Indian Journal of Dental Research, 20(2), 190. https://doi.org/10.4103/0970-9290.52901.

Brancher, J. A., Spada, P. P., Meger, M. N., Fatturri, A. L., Dalledone, M., Bertoli, F. M. de P., Deeley, K., Scariot, R., Vieira, A. R., Küchler, E. C., \& de Souza, J. F. (2019). The association of genetic polymorphisms in serotonin transporter and catechol-O-methyltransferase on temporomandibular disorders and anxiety in adolescents. Journal of Oral Rehabilitation, 46(7), 597-604. https://doi.org/10.1111/joor.12783.

Bueno, C. H., Pereira, D. D., Pattussi, M. P., Grossi, P. K., \& Grossi, M. L. (2018). Gender differences in temporomandibular disorders in adult populational studies: A systematic review and meta-analysis. Journal of Oral Rehabilitation, 45(9), 720-729. https://doi.org/10.1111/joor.12661.

Campos, J. A. D. B., Carrascosa, A. C., Bonafé, F. S. S., \& Maroco, J. (2014). Severity of temporomandibular disorders in women: Validity and reliability of the Fonseca Anamnestic Index. Brazilian Oral Research, 28(1), 16-21. https://doi.org/10.1590/S1806-83242013005000026.

Chaves, T. C., Oliveira, A. S., \& Grossi, D. B. (2008a). Principais instrumentos para avaliação da disfunção temporomandibular , parte I: índices e questionários ; uma contribuição para a prática clínica e de pesquisa. Fisioterapia e Pesquisa, 15(1), 92-100.

Chaves, T. C., Oliveira, A. S., \& Grossi, D. B. (2008b). Principais instrumentos para avaliação da disfunção temporomandibular , Parte II : critérios diagnósticos; uma contribuição para a prática clínica e de pesquisa Main instruments for assessing temporomandibular disorders , part II : diagnostic criteria.Fisioterapia e Pesquisa, 15(1), 101-106.

Chisnoiu, A. M., Picos, A. M., Popa, S., Chisnoiu, P. D., Lascu, L., Picos, A., \& Chisnoiu, R. (2015). Factors involved in the etiology of temporomandibular disorders - a literature review. Clujul Medical, 88(4), 473-478. https://doi.org/10.15386/cjmed-485.

Costa, Y. M., Koninck, B. P., Elsaraj, S. M., Exposto, F. G., Herrero Babiloni, A., Kapos, F. P., Sharma, S., \& Shimada, A. (2021). Orofacial pain education in dentistry: A path to improving patient care and reducing the population burden of chronic pain. Journal of Dental Education, 85(3), 349-358. https://doi.org/10.1002/jdd.12461.

De Leeuw, R., \& Klasser, G. (2013). Orofacial pain: guidelines for assessment, diagnosis, and management (5th ed.).Chicago, U.S.A.: Quintessence.

Figueiredo, N., \& Goes, P. S. A. de. (2009). Construção da atenção secundária em saúde bucal: um estudo sobre os Centros de Especialidades Odontológicas em Pernambuco, Brasil. Cadernos de Saúde Pública, 25(2), 259-267. https://doi.org/10.1590/s0102-311x2009000200004. 
Fillingim, R. B., Ohrbach, R., Greenspan, J. D., Knott, C., Dubner, R., Bair, E., Baraian, C., Mack, N., Gary, D., \& Maixner, W. (2013). Psychological Factors Associated with Development of TMD: the OPPERA Prospective Cohort Study. The journal of pain14(12), 75-90. https://doi.org/10.1016/j.jpain.2013.06.009.Psychological.

Freitas, C. H. S. M., Lemos, G. A., Pessoa, T. R. R. F., Araujo, M. F., \& Forte, F. D. S. (2016). Atenção em saúde bucal: avaliação dos centros de especialidades odontológicas da Paraíba. Saúde Em Debate, 40(108), 131-143. https://doi.org/10.1590/0103-1104-20161080011.

Gonçalves, D. A. D. G., Dal Fabbro, A. L., Campos, J. A. D. B., Bigal, M. E., \& Speciali, J. G. (2010). Symptoms of temporomandibular disorders in the population: an epidemiological study. Journal of Orofacial Pain, 24(3), 270-278.

Guan, G., Kerins, C. C., Bellinger, L. L., \& Kramer, P. R. (2005). Estrogenic effect on swelling and monocytic receptor expression in an arthritic temporomandibular joint model. Journal of Steroid Biochemistry and Molecular Biology, 97(3), 241-250. https://doi.org/10.1016/j.jsbmb.2005.05.013.

Hornung, R. S., Benton, W. L., Tongkhuya, S., Uphouse, L., Kramer, P. R., \& Averitt, D. L. (2020). Progesterone and Allopregnanolone Rapidly Attenuate Estrogen-Associated Mechanical Allodynia in Rats with Persistent Temporomandibular Joint Inflammation. Frontiers in Integrative Neuroscience, 14(26), 116. https://doi.org/10.3389/fnint.2020.00026.

Jimenez-Silva, A., Pena-Duran, C., Tobar-Reyes, J., \& Frugone-Zambra, R. (2017). Sleep and awake bruxism in adults and its relationship with temporomandibular disorders: A systematic review from 2003 to 2014. Acta Odontologica Scandinavica, 75(1), 36-58. https://doi.org/10.1080/00016357.2016.1247465.

Lemos, G. A., Moreira, V. G., Forte, F. D. S., Beltrão, R. T., \& Batista, A. U. D. (2015). Correlation between signs and symptoms of Temporomandibular Disorders (TMD) and severity of malocclusion. Revista de Odontologia da UNESP, 44(3), 175-180. https://doi.org/http://dx.doi.org/10.1590/1807-2577.1084

Lemos, G. A., Paulino, M. R., Forte, F. D. S., Beltrão, R. T. S., Batista, A. U. D. (2015). Influence of temporomandibular disorder presence and severity on oral health-related quality of life. Revista Dor, 16(1), 10-14. https://doi.org/10.5935/1806-0013.20150003.

Lipp, M. E. N. (2005). Inventário de sintomas de stress para adultos de Lipp (3th ed.). Casa do Psicólogo.

List, T., \& Jensen, R. H. (2017). Temporomandibular disorders: Old ideas and new concepts. Cephalalgia, 37(7), 692-704. https://doi.org/10.1177/0333102416686302.

Lobbezoo, F., Ahlberg, J., Glaros, A. G., Kato, T., Koyano, K., Lavigne, G. J., de Leeuw, R., Manfredini, D., Svensson, P., \& Winocur, E. (2013). Bruxism defined and graded: an international consensus. Journal of Oral Rehabilitation, 40(1), 2-4. https://doi.org/10.1111/joor.12011.

Lobbezoo, F., Ahlberg, J., Raphael, K. G., Wetselaar, P., Glaros, A. G., Kato, T., Santiago, V., Winocur, E., De Laat, A., De Leeuw, R., Koyano, K., Lavigne, G. J., Svensson, P., \& Manfredini, D. (2018). International consensus on the assessment of bruxism: Report of a work in progress. Journal of Oral Rehabilitation, 45(11), 837-844. https://doi.org/10.1111/joor.12663.

Magri, L. V., Melchior, M. O., Silva, A. M. B. R., Bataglion, C., Mazzetto, M. O., \& Silva, M. A. M. R. (2018). Profile of a temporomandibular dysfunction and orofacial pain service of a Brazilian public university: what has changed in 10 years? Retrospective study. Brazilian Journal Of Pain, 1(3), 236-240. https://doi.org/10.5935/2595-0118.20180046.

Manfredini, D., Landi, N., Romagnoli, M., Cantini, E., \& Bosco, M. (2003). Etiopathogenesis of parafunctional habits of the stomatognathic system. Minerva Stomatologica, 52(7-8), 339-349.

Medeiros, S. P., Batista, A. U. D., \& Forte, F. D. S. (2011). Prevalência de sintomas de disfunção temporomandibular e hábitos parafuncionais em estudantes universitários. Revista Gaúcha de Odontologia, 59(2), 201-208.

Melo, A. C. R., Forte, F. D. S., Barbosa, G. A. S., \& Batista, A. U. D. (2020). Disfunção Temporomandibular e Dor Orofacial: classificação, epidemiologia, importância do diagnóstico e implicações para o Sistema Único de Saúde (SUS). In R. D. Castro \& A. U. D. Batista (Eds.), Evidências científicas e práticas clínicas odontológicas no âmbito do Sistema Único de Saúde (1th ed., pp. 323-346). João Pessoa: Editora UFPB.

Mello, V. V. C., Barbosa, A. C. S., Morais, M. P. L. A., Gomes, S. G. F., Vasconcelos, M. M. V. B., \& Caldas Júnior, A. F. (2014). Temporomandibular disorders in a sample population of the Brazilian Northeast. Brazilian Dental Journal, 25(5), 442-446. https://doi.org/10.1590/0103-6440201302250.

Michelotti, A., Rongo, R., D’Antò, V., \& Bucci, R. (2020). Occlusion, orthodontics, and temporomandibular disorders: Cutting edge of the current evidence. Journal of the World Federation of Orthodontists, 9(3), S15-S18. https://doi.org/10.1016/j.ejwf.2020.08.003.

Moura, W. P., Silva, P. L. P., Lemos, G. A., Bonan, P. R. F., Montenegro, R. V., \& Batista, A. U. D. (2017). Retrospective review of patients referred to a temporomandibular dysfunction care setting of a Brazilian public university. Revista Dor, 18(2), 128-134. https://doi.org/10.5935/1806-0013.20170026.

Ögren, M., Fältmars, C., Lund, B., \& Holmlund, A. (2012). Hypermobility and trauma as etiologic factors in patients with disc derangements of the temporomandibular joint. International Journal of Oral and Maxillofacial Surgery, 41(9), 1046-1050. https://doi.org/10.1016/j.ijom.2012.02.024.

Okeson, J. P. (2013). Tratamento das desordens Temporomandibulares e Oclusão (7th ed.). - Rio de Janeiro, Brasil: Elsevier.

Oliveira, A. S., Dias, E. M., Contato, R. G., \& Berzin, F. (2006). Prevalence study of signs and symptoms of temporomandibular disorder in Brazilian college students. Brazilian Oral Research, 20(1), 3-7. https://doi.org/10.1590/s1806-83242006000100002.

Oliveira, C. B., Lima, J. A. S., Silva, P. L. P., Forte, F. D. S., Bonan, P. R. F., \& Batista, A. U. D. (2016). Temporomandibular disorders and oral habits in high-school adolescents: a public health issue? RGO - Revista Gaúcha de Odontologia, 64(1), 8-16. https://doi.org/10.1590/1981-863720160001000013054.

Paduano, S., Bucci, R., Rongo, R., Silva, R., \& Michelotti, A. (2020). Prevalence of temporomandibular disorders and oral parafunctions in adolescents from public schools in Southern Italy. Cranio: Journal of Craniomandibular Practice, 38(6), 370-375. https://doi.org/10.1080/08869634.2018.1556893. 
Paulino, M. R., Moreira, V. G., Lemos, G. A., Silva, P. L. P., Bonan, P. R. F., \& Batista, A. U. D. (2018). Prevalence of signs and symptoms of temporomandibular disorders in college preparatory students: Associations with emotional factors, parafunctional habits, and impact on quality of life. Ciencia e Saude Coletiva, 23(1). https://doi.org/10.1590/1413-81232018231.18952015.

Pereira-Junior, F. J., \& Gonçalves, D. A. G. (2020). Diagnostic Criteria for Temporomandibular Disorders: Assessment Instruments (Portuguese). International Network for Orofacial Pain and Related Disorders Methodology. https://ubwp.buffalo.edu/rdc-tmdinternational/tmd-assessmentdiagnosis/dctmd/dc-tmd-translations/.

Pigozzi, L. B., Pereira, D. D., Pattussi, M. P., Moret-Tatay, C., Irigaray, T. Q., Weber, J. B. B., Grossi, P. K., \& Grossi, M. L. (2021). Quality of life in young and middle age adult temporomandibular disorders patients and asymptomatic subjects: a systematic review and meta-analysis. Health and Quality of Life Outcomes, 19(1), 83. https://doi.org/10.1186/s12955-021-01727-7.

Pires, P. F., Castro, E. M., Pelai, E. B., Arruda, A. B. C., \& Rodrigues-Bigaton, D. (2018). Analysis of the accuracy and reliability of the Short-Form Fonseca Anamnestic Index in the diagnosis of myogenous temporomandibular disorder in women. Brazilian Journal of Physical Therapy, 22(4), 276-282. https://doi.org/10.1016/j.bjpt.2018.02.003.

Progiante, P., Pattussi, M., Lawrence, H., Goya, S., Grossi, P., \& Grossi, M. (2015). Prevalence of Temporomandibular Disorders in an Adult Brazilian Community Population Using the Research Diagnostic Criteria (Axes I and II) for Temporomandibular Disorders (The Maringá Study). The International Journal of Prosthodontics, 28(6), 600-609. https://doi.org/10.11607/ijp.4026.

Reis, L. O., Ribeiro, R. A., Martins, C. C., \& Devito, K. L. (2019). Association between bruxism and temporomandibular disorders in children: A systematic review and meta-analysis. International Journal of Paediatric Dentistry, 29(5), 585-595. https://doi.org/10.1111/ipd.12496.

Sánchez-Torrelo, C. M., Zagalaz-Anula, N., Alonso-Royo, R., Ibáñez-Vera, A. J., Collantes, J. L., Rodríguez-Almagro, D., Obrero-Gaitán, E., \& Lomas-Vega, R. (2020). Transcultural Adaptation and Validation of the Fonseca Anamnestic Index in a Spanish Population with Temporomandibular Disorders. Journal of Clinical Medicine, 9(10), 1-11. https://doi.org/10.3390/JCM9103230.

Schiffman, E., Ohrbach, R., Truelove, E., Look, J., Anderson, G., Goulet, J.-P., List, T., Svensson, P., Gonzalez, Y., Lobbezoo, F., Michelotti, A., Brooks, S. L., Ceusters, W., Drangsholt, M., Ettlin, D., Gaul, C., Goldberg, L. J., Haythornthwaite, J. A., Hollender, L., ... Dworkin, S. F. (2014). Diagnostic Criteria for Temporomandibular Disorders (DC/TMD) for Clinical and Research Applications: Recommendations of the International RDC/TMD Consortium Network* and Orofacial Pain Special Interest Group†. Journal of Oral \& Facial Pain and Headache, 28(1), 6-27. https://doi.org/10.11607/jop.1151.

Tay, K. J., Yap, A. U. J., Wong, J. C. M., Tan, K. B. C., \& Allen, P. F. (2019). Associations between symptoms of temporomandibular disorders, quality of life and psychological states in Asian Military Personnel. Journal of Oral Rehabilitation, 46(4), 330-339. https://doi.org/10.1111/joor.12751.

The jamovi project (2021). jamovi (Version 1.6) [Computer Software]. Retrieved June 20, 2021, from https://www.jamovi.org.

Tormes, A. K. M., Lemos, G. A., Silva, P. L. P., Forte, F. D. S., Sousa, F. B., Araujo, D. N., \& Batista, A. U. D. (2020). Temporomandibular disorders: knowledge , competency, and attitudes of predoctoral dental students. Cranio:The Journal of Craniomandibular Practice, Online ahe, 1-9. https://doi.org/10.1080/08869634.2020.1812816.

Valesan, L. F., Da-Cas, C. D., Réus, J. C., Denardin, A. C. S., Garanhani, R. R., Bonotto, D., Januzzi, E., \& de Souza, B. D. M. (2021). Prevalence of temporomandibular joint disorders: a systematic review and meta-analysis. Clinical Oral Investigations, 25(2), 441-453. https://doi.org/10.1007/s00784-02003710-w.

Wagner, B. A., \& Filho, P. F. M. (2018). Painful temporomandibular disorder, sleep Bruxism, anxiety symptoms and subjective sleep quality among military firefighters with frequent episodic tension-type headache. A controlled study. Arquivos de Neuro-Psiquiatria, 76(6), 387-392. https://doi.org/10.1590/0004$282 \times 20180043$

Yap, A. U., \& Natu, V. P. (2020). Inter-relationships between pain-related temporomandibular disorders, somatic and psychological symptoms in Asian youths. Journal of Oral Rehabilitation, 47(9), 1077-1083. https://doi.org/10.1111/joor.13033. 